\title{
Evaluation der Ösophagoskopie im Staging und Restaging von Kopf-Hals-Plattenepithelkarzinomen
}

\section{Evaluation of oesophagoscopy for staging and restaging of head and neck squamous cell carcinoma}

Autoren

Lena Weber ${ }^{1}$, Oliver Blachutzik ${ }^{2}$, Veronika Vielsmeier ${ }^{1}$, Kornelia Andorfer $^{1}$, Christoph Matthias ${ }^{2}$, Julian Künzel $^{1}$

Institute

1 Klinik und Poliklinik für Hals-Nasen-Ohrenheilkunde, Universitätsklinikum Regensburg, Germany

2 Hals-Nasen-Ohrenklinik und Poliklinik, JohannesGutenberg-Universität Mainz, Germany

Schlüsselwörter

Ösophagoskopie, Panendoskopie, Zweittumoren, Staging, Kopf- und Halskarzinome

Key words

panendoscopy, secondary primary, staging, head and neck cancer, oesophagoscopy

eingereicht 26.04.2020

akzeptiert 08.06.2020

online publiziert 26.06 .2020

\section{Bibliografie}

Laryngo-Rhino-Otol 2021; 100: 111-119

DOI 10.1055/a-1197-6978

ISSN 0935-8943

(C) 2020. Thieme. All rights reserved.

Georg Thieme Verlag KG, Rüdigerstraße 14,

70469 Stuttgart, Germany

Korrespondenzadresse

PD Dr. Julian Künzel

Klinik und Poliklinik für Hals-Nasen-Ohrenheilkunde,

Universitätsklinikum Regensburg, Franz-Josef-Strauß-Allee 11, 93053 Regensburg, Germany

Tel.: ++ 49/941/9440

Fax: $++49 / 941 / 9440$

julian.kuenzel@ukr.de

\section{ZUSAMMENFASSUNG}

Hintergrund Die Ösophagoskopie als Teil der Panendoskopie ist ein wichtiger Bestandteil der Diagnostik und Nachsorge von Patienten mit Kopf-Hals-Plattenepithelkarzinomen (HNSCC). Zweitkarzinome haben gravierenden Einfluss auf die Therapie der Primärerkrankung. Diese Arbeit soll prüfen, ob die routinemäßige Ösophagoskopie gerechtfertigt ist oder ob prädiktive Faktoren eine Selektion von Patienten mit erhöhtem Risikoprofil erlauben.
Material und Methoden In einem systematischen Review wurden die Inzidenz von Zweitkarzinomen des Ösophagus und die Komplikationsrate der Ösophagoskopie erfasst. Eine retrospektive Auswertung erfolgte am eigenen Patientenkollektiv. Zur Evaluation der gängigen Praxis an deutschen HNOKliniken wurde eine Umfrage durchgeführt.

Ergebnisse 1053 Ösophagoskopien bei 800 Patienten mit HNSCC wurden ausgewertet. Es traten 7 (0,9\%) synchrone Zweittumoren auf. In 253 Kontroll-Ösophagoskopien wurden 5 (2\%) metachrone Zweittumoren detektiert. In einem Fall $(0,1 \%)$ kam es zu einer iatrogenen Perforation. Ein Zusammenhang zwischen Risikofaktoren (Noxen, Lokalisation Primärtumor etc.) und der Inzidenz von Zweitkarzinomen wurde nicht nachgewiesen. Das Literaturreview ergab eine mittlere gewichtete Inzidenz der Zweitkarzinome für Europa/USA von $1,8 \%$ und $4,1 \%$ für Asien sowie eine Rate der iatrogenen Ösophagusperforation von 0-0,2\%. Die Umfrage zeigte eine routinemäßige Durchführung der Ösophagoskopie im Staging (100\%), in der Nachsorge in 65,3\%.

Schlussfolgerungen Die Ösophagoskopie ist ein sicheres Verfahren zum Ausschluss eines Zweitkarzinoms des Ösophagus. Um eine Patientenselektion nach entsprechendem Risikoprofil - insbesondere in der Tumornachsorge - zu ermöglichen, sind prospektive multizentrische Studien erforderlich.

\section{ABSTRACT}

Objective Oesophagoscopy is important in diagnostic and follow up investigation in patients with head and neck squamous cell carcinoma (HNSCC). Second primary malignancies of the oesophagus have major impact on therapy of the primary tumour. Considering the low incidence of oesophageal second primaries and the serious complication of oesophageal perforation routine oesophagoscopy is being discussed. Material and Methods Incidence of oesophageal second primaries and complication rates in oesophagoscopy were identified in a systematic review. A retrospective analysis was performed in our own patient collective. To evaluate the current practice at German ENT Clinics a survey was conducted.

Results 1053 oesophagoscopies in 800 patients were analysed. In 800 patients seven $(0.9 \%)$ synchronous secondary malignancies of the oesophagus occurred. In 253 follow up oesophagoscopies five (2\%) metachronous secondary malignancies were discovered. 14 (1.3\%) complications were de- 
tected; oesophageal perforation was only detected in one case $(0.1 \%)$. There was no association of certain risk factors with the incidence of secondary malignancies. The review of literature showed an incidence of secondary malignancies for Europe/USA of $1.8 \%$ and for Asia of $4.1 \%$. Incidence of oesophageal perforation was $0-0.2 \%$. Survey results showed routine oesophagoscopy in staging (100\%) and regularly in follow up $(65.3 \%)$.
Conclusions Oesophagoscopy is a convenient method to detect secondary malignancies of the oesophagus. To allow a selection of patients developing secondary malignancies according to risk profiles further prospective multicentre studies are required.

\section{Einleitung}

Die Ösophagoskopie als Teil der Panendoskopie ist traditionell ein wichtiger Bestandteil der Diagnostik und Nachsorge von Patienten mit Plattenepithelkarzinomen im Kopf-Hals-Bereich (HNSCC). Zweitkarzinome im Ösophagus haben gravierenden Einfluss auf die Therapie der Primärerkrankung und führen zu einer deutlich reduzierten Überlebensrate [1]. Dies macht eine frühzeitige und gründliche Detektion notwendig. Tritt ein Zweitkarzinom innerhalb von 6 Monaten nach Diagnosestellung eines HNSCC auf, wird es als synchron bezeichnet, zu jedem späteren Zeitpunkt als metachron [2].

Die Inzidenzen von Zweitkarzinomen im Ösophagus variieren regional erheblich. In den USA und Europa wird die Inzidenz von synchronen Zweitkarzinomen mit 2-3\% [3-5] angegeben, in asiatischen Ländern hingegen mit 4-7\% [6, 7]. Wesentliche Risikofaktoren für die Entstehung von Sekundärtumoren sind ein persistierender Nikotin- und Alkoholabusus [3]. Die Kombination dieser Noxen mit dem Verzehr von Betelnüssen wird für die erhöhte Inzidenz in asiatischen Ländern verantwortlich gemacht [8]. Bisher wurde kein Einfluss des Stadiums des Primärtumors auf die Inzidenz nachgewiesen, jedoch wird diskutiert, ob abhängig von der Lokalisation des Primärtumors ein erhöhtes Risiko für Zweitmalignome bestehen könnte [9]. Patienten mit HPV-assoziierten Tumoren weisen häufig nicht das typische Risikoprofil auf. Dies wirft die Frage auf, ob bei Fehlen von Risikofaktoren die Wahrscheinlichkeit für Zweitkarzinome so niedrig ist, dass im Rahmen des Stagings bei diesen Patienten auf eine Ösophagoskopie verzichtet werden kann. Verlässliche Methoden zur Selektion der zu untersuchenden Patienten sind bisher nicht etabliert.

Das für die Ösophagoskopie verwendete Endoskop kann starr oder flexibel sein. Beide Verfahren bergen Risiken für die Patienten. Schäden an den Zähnen und Schleimhautverletzungen zählen dabei zu den harmloseren Komplikationen. Die Ösophagusperforation mit der Gefahr einer Mediastinitis stellt die gravierendste Komplikation dar. In Bezug auf diagnostische Ösophagoskopien liegt die Inzidenz für Perforationen deutlich unter $1 \%$ [10]. Dabei wird die Mortalitätsrate bei einer ösophagealen Perforation mit 15-20\% angegeben [11].

Ziel dieser Arbeit ist es zu prüfen, ob die starre Ösophagoskopie als Instrument der routinemäßigen Diagnostik und Nachsorge von HNSCC gerechtfertigt ist oder ob prädiktive Faktoren eine Selektion von Patienten mit erhöhtem Risikoprofil erlauben. Wir führten ein systematisches Review zur Ermittlung der Inzidenz von Zweitkarzinomen und der Komplikationsrate der Ösophago- skopie durch. Darüber hinaus haben wir unser eigenes Patientenkollektiv diesbezüglich retrospektiv ausgewertet. Zur Evaluation der gängigen Praxis der Durchführung der Ösophagoskopie im Rahmen der Panendoskopie wurde eine Umfrage mittels Fragebogen an deutschen HNO-Kliniken erstellt.

\section{Material und Methode}

Die Studie wurde von der Ethikkommission der Landesärztekammer Rheinland-Pfalz genehmigt (2019-14060).

\section{Patientenkollektiv}

Das Kollektiv dieser retrospektiven Studie setzt sich aus Patienten einer deutschen HNO-Universitätsklinik zusammen. Patienten, bei denen zwischen 2008 und 2018 aufgrund eines HNSCC im Rahmen einer Panendoskopie eine Ösophagoskopie durchgeführt wurde, konnten in die Studie eingeschlossen werden. KontrollPanendoskopien wurden vorwiegend bei Patienten mit nichtchirurgischer Primärtherapie oder bei Rezidiv-Verdacht durchgeführt. Bei erhöhtem Operationsrisiko wurde ebenfalls eher auf eine Kontroll-Panendoskopie bei unauffälligem Spiegelbefund und unauffälliger Bildgebung im Restaging verzichtet. Zur Identifikation aller Patienten, auf die die obengenannten Kriterien zutrafen, wurde eine Datenbankabfrage (SAP-SE, Walldorf/Deutschland) mit Operations- und Prozedurenschlüssel (OPS)-Codes durchgeführt. Verwendet wurden die Codes der flexiblen Ösophagoskopie (1-630.0), der starren Ösophagoskopie (1-630.1), der Ösophagogastroskopie (1-631), der Ösophagogastroduodenoskopie (1-632) sowie der Kombination der Panendoskopie bestehend aus flexibler Tracheobronchoskopie (1-620.0), starrer Ösophagoskopie (1-630.1), diagnostischer Laryngoskopie (1-610.0) und diagnostischer Pharyngoskopie (1-611.0). Zu den Patienten wurden Geburtsdatum, Geschlecht, Body-Mass-Index, Nikotinkonsum in pack years, Ausprägung des Alkoholkonsums (anamnestische Angaben, kategorisiert nach Anzahl des Konsums pro Woche), bisherige Tumoren im Aerodigestivtrakt (innerhalb der letzten 5 Jahre) und deren Lokalisation, Lokalisation des Primarius, klinische Befunde im Ösophagus, bereits durchgeführte Radiatio, Datum und Art der Ösophagoskopie, Biopsie-Befunde, das Vorhandensein eines synchronen Tumors, Komplikationen und Art der Komplikationen, eine temporär eingesetzte Magensonde sowie das Vorhandensein eines metachronen Tumors strukturiert erfasst. 


\section{Literaturrecherche}

Zur Evaluation der bestehenden Evidenz führten wir eine systematische Literaturabfrage nach den Prisma-Kriterien (www.prismastatement.org) durch. Die Abfrage erfolgte von September bis Oktober 2018 über die Datenbank PubMed (NLM). Folgende MeSH- Begriffe wurden abgefragt: „esophagoscopy OR flexible esophagoscopy AND head and neck cancer AND squamous cell carcinoma”, „esophagoscopy AND head and neck cancer AND second primary” und „panendoscopy AND head and neck cancer”. Zudem wurden die Filter „only humans“, „english (language)“ und „abstract available“ angewendet.

Auswahlkriterium geeigneter Arbeiten war die initiale Diagnose eines HNSCC und die Durchführung einer Ösophagoskopie. Der Einschluss in die systematische Übersicht erfolgte für alle Arbeiten, bei denen eine exakte Inzidenz für synchrone Zweitkarzinome im Ösophagus oder eine Inzidenz für Komplikationen im Rahmen der Ösophagoskopie angegeben wurde.

\section{Umfrage}

Es erfolgte eine deutschlandweite Umfrage an Hals-Nasen-Ohrenkliniken, um den aktuellen Stellenwert der Ösophagoskopie als diagnostisches Verfahren bei Kopf- und Hals-Tumorpatienten in der klinischen Praxis einzuordnen. Die E-Mail-Adressen der Klinikleitungen wurden uns für den Zweck dieser Umfrage freundlicherweise von der Geschäftsstelle der Deutschen Gesellschaft für Hals-Nasen-Ohrenheilkunde, Kopf- und Halschirurgie zur Verfügung gestellt.

Der Fragebogen beinhaltete 5 Fragen:

a) „Wird die Ösophagoskopie standardmäßig im Staging von Kopf-Hals-Tumorpatienten angewendet?“

b) „Welches Endoskop verwenden Sie zur Ösophagoskopie im Rahmen der Panendoskopie?“

Auswahl: flexibel oder starr

c) „Halten Sie die Ösophagoskopie im Primärstaging für sinnvoll?“

d) „Führen Sie eine Kontroll-Ösophagoskopie standardmäßig in der Nachsorge durch?“

e) „Halten Sie die Ösophagoskopie im Restaging für sinnvoll?“

Weiterhin bestand die Möglichkeit für einen Freitextkommentar.

\section{Statistik}

Die erfassten Daten wurden mit Microsoft Excel für Mac (Microsoft-Excel Version 16.24, Microsoft, Redmond/ Vereinigte Staaten) sowie mit IBM SPSS Statistic Subscription (Version 23, IBM, Armonk/ Vereinigte Staaten) für Mac statistisch ausgewertet (univariate Analysen, exakter Fisher-Test, Chi-Quadrat-Test). Ein p-Wert $<0,05$ wurde als statistisch auffällig gewertet.

\section{Ergebnisse}

\section{Patientenkollektiv}

Insgesamt wurden 1053 Ösophagoskopien bei 800 Patienten ausgewertet. Dieses Kollektiv setzte sich aus 624 Männern (78\%) und 176 Frauen zusammen (22\%). Das Durchschnittsalter zum Zeit-

- Tab. 1 Häufigkeit und Menge an Nikotinkonsum gruppiert nach Anzahl der pack years (py), n (\%).

\section{pack years}

0

$1-20$

$21-40$

$>40$

keine Angabe

Tab. 2 Ausprägung des Alkoholkonsums, n (\%).
Alkoholkonsum$$
\text { nie }
$$

nie

selten (bis 1-mal/Woche)

moderat (1-2-mal/Woche)

stark (>2-mal/Woche)

keine Angabe
Anzahl (relative Häufigkeit in \%)

$173(21,6 \%)$

$83(10,4 \%)$

$243(30,4 \%)$

$232(29 \%)$

$69(8,6 \%)$ punkt der Ösophagoskopie betrug 63,9 Jahre. Unter den Patienten waren 558 Raucher (69,8\%), 173 Nichtraucher (21,6\%), bei 69 Patienten $(8,6 \%)$ fehlten entsprechende Angaben dazu in der Patientenakte. Die Anzahl der pack years ist $>$ Tab. 1 zu entnehmen. 445 (55,6\%) Patienten gaben einen regelmäßigen Alkoholkonsum an. Dagegen verneinten 69 (8,6\%) Patienten einen Alkoholkonsum, während 286 (35,8\%) keine Angaben dazu machten bzw. keine Angaben dokumentiert waren. Die Ausprägung des Alkoholkonsums ist in Tab. 2 dargestellt. Die Existenz eines Tumors im Kopf-Hals-Bereich in der medizinischen Vorgeschichte wurde nach Durchsicht der Anamnesen in 102 Fällen (12,8\%) bestätigt und in 698 Fällen $(87,2 \%)$ ausgeschlossen. 66 (8,2\%) Patienten hatten zum Zeitpunkt der Ösophagoskopie bereits eine Strahlentherapie in der Anamnese. Abb. 1 zeigt, dass Mundhöhlen-, Oropharynx- und Larynxkarzinome mit jeweils ca. 25 \% die häufigsten Lokalisationen der Primärtumoren in diesem Kollektiv waren.

Von den insgesamt 1053 durchgeführten Ösophagoskopien wurden 22 (1,8\%) mit einem flexiblen und 1031 (98,2\%) mit einem starren Endoskop durchgeführt. Bei 34 (4,3\%) Patienten wurden Biopsien aus dem Ösophagus zur Abklärung klinisch auffälliger Befunde genommen. Der histologische Befund ergab hierbei in 10 Fällen unauffällige Schleimhaut, in 7 Fällen ein Plattenepithelkarzinom, in jeweils 6 Fällen eine chronische Entzündung oder ektope Magenschleimhaut und Barett-Ösophagus in 4 Fällen. Ein Patient präsentierte eine Soor-Ösophagitis. Unter den 800 Patienten traten somit insgesamt 7 (0,9\%) histologisch gesicherte synchrone Zweittumoren des Ösophagus auf.

Es wurden 14 Komplikationen (1,3\%) dokumentiert, wobei es sich in nur einem Fall $(0,1 \%)$ um eine Perforation des Ösophagus handelte. Die restlichen 13 Komplikationen waren geringfügige 


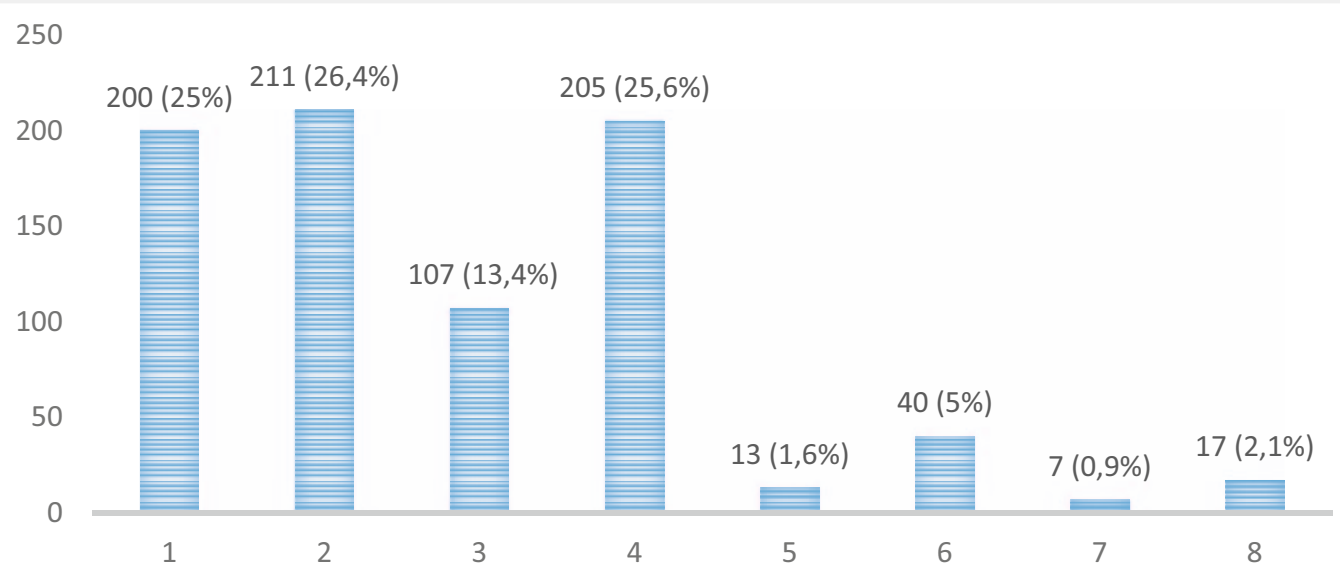

- Abb. 1 Lokalisation und Häufigkeit der Primärtumoren. 1-Mundhöhle; 2-Oropharynx; 3-Hypopharynx; 4-Larynx; 5-Nasopharynx; 6-Cancer unknown primary (CUP); 7-andere; 8-multiple Tumoren.

Komplikationen wie mukosale Abrasionen, iatrogen induzierte Blutungen und ein dentales Trauma. Keine der aufgetretenen Komplikationen hatte einen letalen Ausgang. Bei 33 (4,1\%) Patienten wurde während oder nach Durchführung der Ösophagoskopie eine temporäre nasogastrale Sonde eingesetzt. Gründe hierfür waren temporäre schwellungsbedingte Schluckunfähigkeit $(n=30)$ sowie in einem Fall eine Perforation des Ösophagus. In 2 Fällen war in der Patientenakte keine Begründung zu ermitteln.

Insgesamt 253 (31,6\%) der 800 Probanden erhielten im Verlauf der Nachsorge eine Kontroll-Ösophagoskopie. Dabei wurden insgesamt 5 (2\%) metachrone Zweittumoren entdeckt. Diese 5 Zweittumoren wurden nach 9, 12, 18, 36 und 60 Monaten diagnostiziert.

Die Diagnose eines Zweitkarzinoms im Ösophagus (synchron und metachron) führte in 7 Fällen zu einer Ergänzung des kurativen Therapieansatzes, in den anderen 5 Fällen lag eine palliative Situation vor.

Die Merkmale „Geschlecht“, „Rauchen“, „pack years“, „Ausprägung des Alkoholkonsums“, „Lokalisation des Primärtumors“ und „klinischer Befund im Ösophagus“ zeigten statistisch keinen Zusammenhang mit dem Auftreten von Zweitmalignomen $(p>0,05)$. Für die Merkmale „Tumor des oberen Aerodigestivtrakts in der Vorgeschichte“ und „metachroner Tumor“ lag der ermittelte $p$-Wert bei 0,032 . Folglich zeigte sich hier ein statistisch auffälliger Wert.

In Bezug auf die Häufigkeit von Komplikationen gab es unter Prüfung unterschiedlichster Merkmale und deren Kombinationen keine statistischen Auffälligkeiten.

\section{Literaturrecherche}

Zur Ermittlung der bestehenden Evidenz ergab das nach den Prisma-Kriterien durchgeführte systematische Literaturreview eine Anzahl von 887 Arbeiten, die in die Vorauswahl aufgenommen wurden. Die Prüfung auf Eignung der jeweiligen Arbeiten sowie deren Einschluss in die Auswertung ist in $\mathbf{A b b} \mathbf{2}$ als Flussdiagramm veranschaulicht.
In Bezug auf die Inzidenzen für synchrone Zweittumoren im Ösophagus in Europa und den USA ergaben sich Werte zwischen 0 und 11,5\%, mit einem Mittelwert von 2,2\%. Unter Berücksichtigung der genannten Fallzahlen errechnete sich ein gewichteter Mittelwert von 1,8\%. Betrachtet man die Zahlen der Studien aus asiatischen Ländern, so ließen sich dort Werte zwischen 0,8 und 26,9\% finden. Der Mittelwert hierfür lag bei 12,1\% und der gewichtete Mittelwert bei 4,1\%.

Die Inzidenz für die schwerste Komplikation, die iatrogene Ösophagusperforation, wurde in den eingeschlossenen Studien mit 0 bis $0,2 \%$ angegeben. > Tab. 3 enthält eine detaillierte Aufstellung aller ausgewerteten Publikationen aus Amerika [4, 1232], Europa [3, 5, 33-39], Australien [40] und Asien [6-9, 41-52].

\section{Umfrage}

Die Rücklaufquote des Umfragebogens lag bei 29,9\% (49/164). Die Frage nach der standardmäßigen Durchführung der Ösophagoskopie im Staging von Kopf-Hals-Tumorpatienten wurde von allen 49 Kollegen mit „Ja“ beantwortet (100\%).

In 69,4\% erfolgt die starre Ösophagoskopie, in 8,2\% eine flexible Spiegelung und in 11 Kliniken (22,4\%) werden beide Methoden angewandt. Die überwiegende Mehrheit (91,8\%) betrachtet die Ösophagoskopie im Primärstaging als sinnvoll und 65,3\% der Befragten führen auch in der Nachsorge regelmäßig eine KontrollÖsophagoskopie durch. Allerdings wird die routinemäßige Kontroll-Ösophagoskopie nur noch von $73,5 \%$ als sinnvoll erachtet.

\section{Diskussion}

Die Panendoskopie ist im Rahmen des Stagings von HNSCC eine etablierte Methode zur histologischen Sicherung des Primärtumors, Erfassung der Größenausdehnung, Einschätzung der Operabilität und Detektion von Zweitkarzinomen. Betrachtet man das Patientenkollektiv dieser Studie, geben $70 \%$ einen regelmäßigen Nikotin- und $56 \%$ einen regelmäßigen Alkoholkonsum an. Nach der Theorie der Feldkanzerisierung wird aufgrund der flächi- 


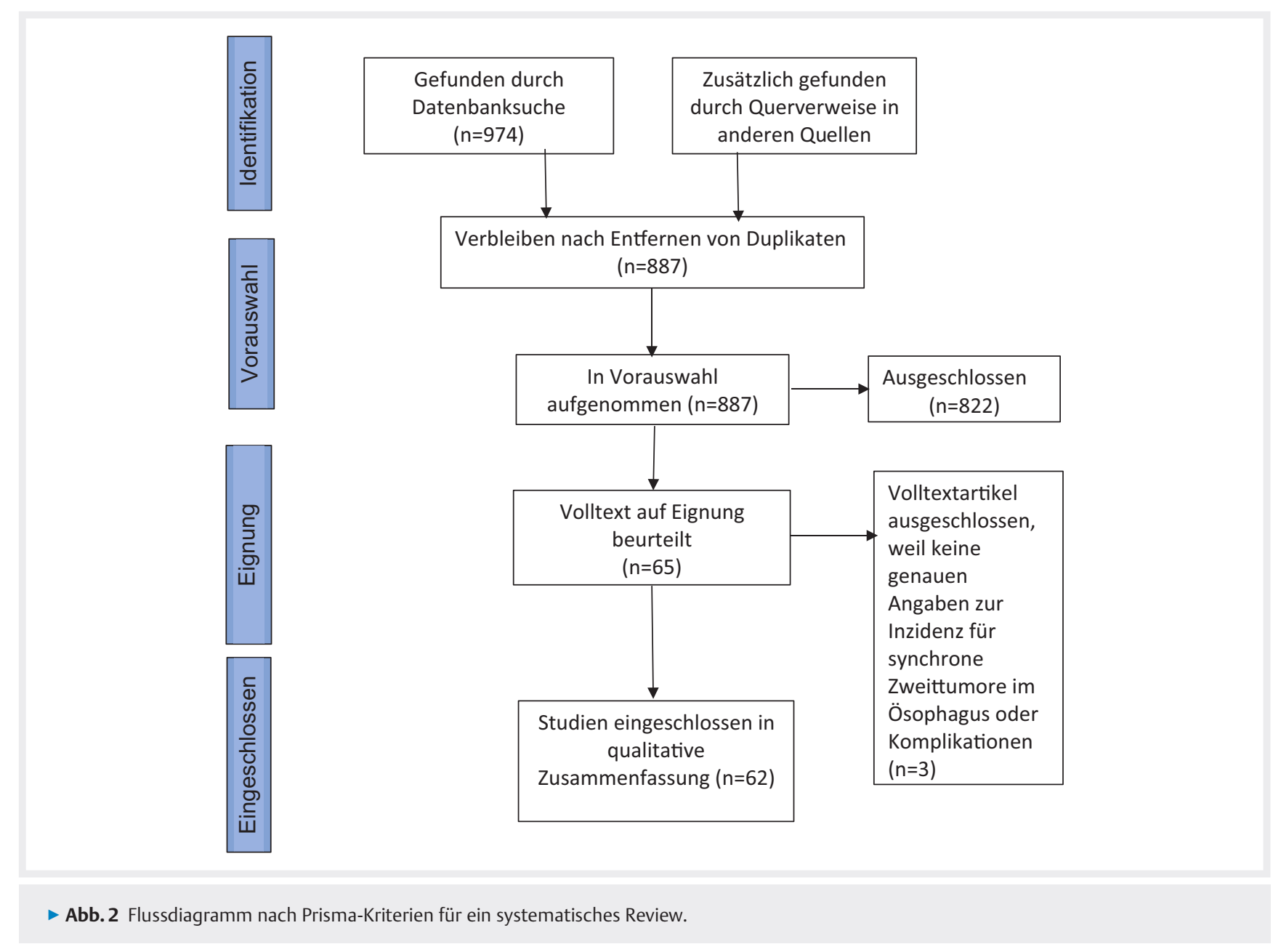

gen Angriffszone dieser Noxen prinzipiell von einem erhöhten Risiko für ein Zweitkarzinom ausgegangen [53, 54]. In diesem monozentrischen Patientenkollektiv zeigte sich eine Rate von $0,9 \%$ für synchrone Zweitkarzinome. In Europa und den USA liegt die Inzidenz hierfür im Mittel bei 1,8\%. Das Auftreten synchroner Zweitkarzinome des Ösophagus ist somit in westlichen Ländern als selten einzustufen, allerdings sind die Auswirkungen auf Therapieansatz, Therapieerfolg und die Überlebensrate der Patienten durchaus gravierend. Die Indikation zur Ösophagoskopie liegt in der rechtzeitigen Erkennung eines asymptomatischen Zweitkarzinoms in einem frühen, noch kurativ therapierbaren Stadium [12]. In asiatischen Ländern ergibt sich mit 4,1 \% eine etwa 2,5-fach höhere Inzidenz für primäre Zweitkarzinome des Ösophagus. Der deutliche Unterschied lässt darauf schließen, dass dort weitere ursächliche Faktoren existieren. Das Konsumverhalten, insbesondere das Kauen von Betelnüssen sowie der Verzehr von gepökeltem Fleisch (karzinogene Nitrosamine), wird als zusätzlicher Risikofaktor vermutet [55].

Die Inzidenz von metachronen Zweitkarzinomen von $2 \%$ in diesem Kollektiv verdeutlicht, dass die Ösophagoskopie auch in der Tumornachsorge ihren Stellenwert hat. Es ist zu berücksichtigen, dass nicht alle Patienten in diesem Kollektiv eine routinemäßige Kontroll-Panendoskopie bzw. Kontroll-Ösophagoskopie erhielten. Dies entspricht einem Selektionsbias. Kuhlin et al. plä- dieren für eine intensive Nachsorge, um die frühe Detektion von Zweitkarzinomen zu sichern [56].

Einige Autoren sehen die routinemäßige Ösophagoskopie kritisch und propagieren eine Selektion von Patienten mit einem gesteigerten Risikopotenzial [31, 32]. Dies gilt beispielsweise bei persistierendem Alkohol- und Nikotinabusus sowie letztlich auch bei klinischen Symptomen wie neu aufgetretener Dysphagie. Unsere Daten ergeben Hinweise, dass bei Zustand nach HNSCC in der Vorgeschichte ein erhöhtes Risiko für metachrone Tumoren besteht. Die Lokalisation des Primärtumors (Oropharynx, Hypopharynx, supra- und transglottisches Larynxkarzinom) könnte nach einer Studie von Su et al. ebenfalls einen Einfluss auf die Inzidenz von synchronen Ösophaguskarzinomen haben [57]. Dieser Zusammenhang konnte in unserer Studie nicht bestätigt werden. Auch Kuhlin et al. konnten weder für die Lokalisation des Primärtumors, noch für Alter, Geschlecht oder TNM-Stadium signifikante Zusammenhänge für die Inzidenz von Zweitkarzinomen identifizieren [56]. Die Literaturdaten erlauben aktuell keine Empfehlung, in welcher Konstellation auf die Ösophagoskopie verzichtet werden kann.

Die Auswertung der Komplikationsraten ergab mit 1,3\% insgesamt einen geringen Wert. Die schwerste Komplikation, die Ösophagusperforation, trat in unserem Kollektiv nur in einem Fall $(0,1 \%)$ auf. Die Literaturrecherche ergab mit $0-0,2 \%$ ebenfalls ge- 
- Tab. 3 Inzidenzen für synchrone Zweitkarzinome im Ösophagus diagnostiziert durch starre oder flexible Ösophagoskopie in Amerika, Europa, Australien und Asien.

\begin{tabular}{|c|c|c|c|c|}
\hline Autor & Zeitraum & Standort & $\begin{array}{l}\text { Inzidenz für synchrone } \\
\text { Tumoren (Anz.|\%) }\end{array}$ & Ösophagusperforation (\%) \\
\hline Weaver et al. [12], 1979 & 1976-1978 & Detroit, Michigan & $3 / 124(2,4 \%)$ & n.a. \\
\hline Cohn, Peppard [13]*, 1980 & 1970-1979 & Detroit, Michigan & $8 / 267(3,0 \%)$ & n.a. \\
\hline Shapsay et al. [14] ${ }^{*}, 1980$ & 1977-1978 & Boston, Massachusetts & $9 / 150(6 \%)$ & n. a. \\
\hline Gluckman et al. [15], 1980 & 1977-1979 & Cincinnati, Ohio & $4 / 259(1,5 \%)$ & n. a. \\
\hline Maisel, Vermeersh [16], 1981 & $1971-1980$ & Minneapolis, Minnesota & $4 / 449(0,9 \%)$ & n. a. \\
\hline McGuirt [17], 1982 & n.a. & $\begin{array}{l}\text { Winston-Salem, } \\
\text { North-Carolina }\end{array}$ & $8 / 100(8 \%)$ & n. a. \\
\hline McGuirt et al. [18], 1982 & n.a. & n. a., USA & $6 / 81(7,4 \%)$ & $0 / 81(0 \%)$ \\
\hline Grossmann et al. [19], 1983 & 1973-1982 & Milwaukee, Wisconsin & $17 / 696(2,4 \%)$ & n. a. \\
\hline Atkins et al. [20], 1984 & 1974-1983 & Philadelphia, Pennsylvania & $3 / 451(0,7 \%)$ & n. a. \\
\hline Leipzig et al. [4], 1985 & 1983-1984 & verschiedene Standorte & $7 / 384(1,8 \%)$ & n. a. \\
\hline Schuller, Fritsch [21], 1986 & n.a. & Columbus, Ohio & $0 / 53(0 \%)$ & $0 / 53(0 \%)$ \\
\hline Grossmann et al. [22], 1987 & 1983-1986 & Milwaukee, Wisconsin & $4 / 254(1,6 \%)$ & n. a. \\
\hline Parker, Hill [23], 1988 & 1984-1985 & Chicago, Illinois & $4 / 208(1,9 \%)$ & 0/208 (0\%) \\
\hline Shaha et al. [24], 1988 & 1982-1986 & Brooklyn, New York & $3 / 140(1,7 \%)$ & n. a. \\
\hline Abemayor et al. [25], 1988 & 1984-1985 & Los Angeles, California & $3 / 150(2 \%)$ & n. a. \\
\hline Grossmann et al. [26]*, 1989 & 1970-1980 & Milwaukee, Wisconsin & $9 / 790(1,1 \%)$ & n. a. \\
\hline Atabek et al. [27] ${ }^{*}, 1990$ & 1980-1986 & Camden, New Jersey & $5 / 501(1 \%)$ & n. a. \\
\hline Contini et al. [33], 1991 & 1986-1988 & Parma, Italy & $3 / 103(2,9 \%)$ & n. a. \\
\hline Dhooge et al. [34], 1996 & 1990-1992 & Gent, Belgien & $0 / 124(0 \%)$ & n. a. \\
\hline Cianfraglia et al. [35]*, 1999 & 1989-1992 & Rom, Italien & $0 / 200(0 \%)$ & n. a. \\
\hline Davidson et al. [28], 2000 & n.a. & Toronto, Canada & $0 / 154(0 \%)$ & $0 / 154(0 \%)$ \\
\hline Stoeckli et al. [5], 2001 & 1990-1995 & Zürich, Schweiz & $4 / 358(1,1 \%)$ & n. a. \\
\hline Scherübl et al. [3], 2002 & $2000-2001$ & Berlin, Deutschland & $4 / 148(2,7 \%)$ & $0 / 148(0 \%)$ \\
\hline Guardiola et al. [36], 2004 & 1989-2000 & Besancon, Frankreich & $10 / 487(2 \%)$ & n. a. \\
\hline Hujala et al. [37], 2005 & 1992-1999 & Turku, Finnland & $0 / 203(0 \%)$ & n. a. \\
\hline Hashimoto et al. [29]*, 2005 & $1995-2000$ & Sao Paulo, Brasilien & $24 / 326(7,3 \%)$ & n. a. \\
\hline Moschler et al. [38], 2006 & 2000-2004 & Kiel, Deutschland & $10 / 87(11,5 \%)$ & n. a. \\
\hline Tsao, Damrose [30], 2010 & 2002-2007 & Stanford, California & $0 / 546(0 \%)$ & $3 / 99(3 \%)$ \\
\hline Rodriguez-Bruno et al. [31], 2010 & $2000-2009$ & San Francisco, California & $0 / 64(0 \%)$ & n. a. \\
\hline Carvalho et al. [39], 2013 & $2008-2012$ & Coimbra, Portugal & $1 / 89(1,1 \%)$ & $0 / 89(0 \%)$ \\
\hline Koo et al. [40], 2015 & n. a. & Melbourne, Australien & $0 / 112(0 \%)$ & n. a. \\
\hline McGarey et al. [32], 2016 & 2004-2012 & Charlottesville, Virginia & $0 / 601(0 \%)$ & $1 / 601(0,2 \%)$ \\
\hline Okumura et al. [6], 1993 & 1987-1991 & Osaka, Japan & $11 / 150(7,3 \%)$ & n. a. \\
\hline Ina et al. [41], 1994 & 1990-1993 & Tokyo, Japan & $8 / 127(6,3 \%)$ & n. a. \\
\hline Muto et al. [42], 2002 & $1992-2000$ & Tokyo, Japan & $54 / 389(13,9 \%)$ & n. a. \\
\hline Chow et al. [9], 2009 & $2000-2006$ & Hongkong, China & $9 / 118(7,6 \%)$ & n. a. \\
\hline Fukuhara et al. [43], 2010 & 2003-2006 & Hiroshima, Japan & $17 / 157(10,8 \%)$ & n. a. \\
\hline Morimoto et al. [44], 2010 & 1999-2006 & Osaka, Japan & $18 / 64(28,1 \%)$ & n. a. \\
\hline Lee et al. [45], 2010 & 2008-2009 & Kaohsiung, Taiwan & $16 / 69(23,2 \%)$ & n. a. \\
\hline Su et al. [7], 2013 & $2007-2010$ & Kaohsiung, Taiwan & $71 / 1592(4,5 \%)$ & n. a. \\
\hline Hung et al. [8], 2013 & 2002-2009 & Taipei, Taiwan & $65 / 2965$ (2,2\%) & n. a. \\
\hline
\end{tabular}


- Tab. 3 (Fortsetzung)

\begin{tabular}{|c|c|c|c|c|}
\hline Autor & Zeitraum & Standort & $\begin{array}{l}\text { Inzidenz für synchrone } \\
\text { Tumoren (Anz.|\%) }\end{array}$ & Ösophagusperforation (\%) \\
\hline Chung et al. [46], 2013 & $2010-2012$ & Taipei, Taiwan & $30 / 129(23,3 \%)$ & n.a. \\
\hline Kim et al. [47], 2014 & 2010-2012 & Seoul, Südkorea & $22 / 308(7,1 \%)$ & n.a. \\
\hline Laohawiriyakamol et al. [48], 2014 & $2009-2011$ & Songkhla, Thailand & $11 / 89(12,4 \%)$ & n.a. \\
\hline Krishnatreya et al. [49], 2015 & 2010-2011 & Indien & $35 / 4184(0,8 \%)$ & n.a. \\
\hline Gong et al. [50], 2016 & 2010-2014 & Seoul, Südkorea & $24 / 458(5,2 \%)$ & n. а. \\
\hline Huang et al. [51], 2016 & $2007-2014$ & Taipei, Taiwan & $36 / 248(14,5 \%)$ & n.a. \\
\hline Ni et al. [52], 2018 & n.a. & Peking, China & $43 / 160(26,9 \%)$ & n.a. \\
\hline
\end{tabular}

ringe Raten an Ösophagusperforationen. Insgesamt stellt die Ösophagoskopie ein sicheres Verfahren mit einem kalkulierbaren Risiko für den Patienten dar.

Die Umfrage ergab, dass in bis zu 30 \% der Kliniken neben der starren Ösophagoskopie auch flexible Spiegelungen durchgeführt werden. Nachteile der starren Ösophagoskopie sind die Notwendigkeit einer Narkose, die Einschränkung bei ungenügender Reklination des Kopfes sowie die Tatsache, dass häufig distal nicht der gesamte Ösophagus dargestellt wird. Je nach Länge des Ösophagoskops wird meist bis ca. $30 \mathrm{~cm}$ ab oberer Zahnreihe untersucht. Vor allem der gastroösophageale Übergang als Lokalisation von Barrett-Metaplasien und Adenokarzinomen bleibt dem Untersucher somit häufig verborgen. Die starre Ösophagoskopie ist allerdings im Rahmen der Panendoskopie relativ schnell, einfach und kostengünstig durchzuführen, bietet optimale Sicht auf die Postkrikoid-Region und den proximalen Ösophagus und erlaubt größere Biopsien. Im Rahmen der Umfrage wurde die Möglichkeit der Bougierung zur Auflösung von postradiogenen Stenosen im Rahmen der starren Ösophagoskopie positiv bewertet. Als Nebenaspekt sei die regelmäßige Durchführung der starren Ösophagoskopie vorteilhaft, um für schwierige Fremdkörperentfernungen genügend Routine zu besitzen. Auch in der Ausbildung der Assistenten ist die starre Ösophagoskopie als wichtige Untersuchungsmethode der Hals-Nasen-Ohrenheilkunde fester Bestandteil.

Flexible Endoskope sind deutlich aufwendiger in der Aufbereitung und kostenintensiv in der Anschaffung. Die flexible Ösophagoskopie ist allerdings in der Regel in Analgosedierung möglich. Dabei können der gastroösophageale Übergang sowie der Magen bis zum Duodenum komplett untersucht werden. Die Erfolgsrate der Biopsien wird in der Literatur für die starre Ösophagoskopie mit 99,3\% und für die flexible Ösophagoskopie mit 80,5\% angegeben [58]. Die S1-Leitlinie Ösophagoskopie erachtet beide Verfahren im Rahmen der Panendoskopie als gleichwertig [59]. Bildgebende Verfahren (CT, MRT, PET/CT) haben als alleinige Screening-Methoden keine ausreichende Sensitivität in der Detektion von Zweitkarzinomen des Ösophagus [22, 36, 60]. Der Nutzen der PET/CT im Staging ist prinzipiell hoch, jedoch aufgrund der Strahlenbelastung und der hohen Kosten sowie der nicht flächendeckenden Verfügbarkeit als Standard kritisch zu bewerten
[61]. Vorläuferläsionen oder Frühstadien von Zweitmalignomen des Ösophagus können mit keinem der gängigen bildgebenden Verfahren ausreichend sicher detektiert werden.

Die Inzidenzen von 1-2 \% für synchrone bzw. metachrone Zweitkarzinome des Ösophagus in diesem Kollektiv zusammen mit den Resultaten der Umfrage bekräftigen den hohen Stellenwert der Ösophagoskopie im Rahmen des Stagings und Restagings von HNSCC. Die Umfrage zeigt, dass die Ösophagoskopie in den meisten HNO-Kliniken in Deutschland standardmäßig im Staging durchgeführt wird. Auch in der Nachsorge von Patienten mit HNSCC führen ca. $65 \%$ der HNO-Kliniken die Ösophagoskopie durch.

\section{Schlussfolgerung}

Der Ausschluss bzw. die Detektion von Zweitmalignomen des Ösophagus im Rahmen des Stagings von HNSCC ist obligat. Dafür ist die Ösophagoskopie als Teil der Panendoskopie ein geeignetes und etabliertes Verfahren. Bildgebende Verfahren ersetzen nicht die endoskopische Untersuchung des Ösophagus. Die Unterschiede in den Komplikationsraten zwischen starrem und flexiblem Endoskop stellen sich so marginal dar, dass keine generelle Empfehlung für eines der beiden Verfahren erfolgen kann. In der Nachsorge erscheint die flexible Ösophagoskopie aufgrund der Durchführbarkeit in Analgosedierung vorteilhaft zu sein.

Die Ergebnisse dieser Studie decken sich hinsichtlich der Inzidenz für synchrone Zweitmalignome und den beschriebenen Komplikationsraten mit den Literaturangaben. Um eine Patientenselektion nach entsprechendem Risikoprofil - insbesondere in der Tumornachsorge - zu ermöglichen, sind prospektive multizentrische Studien erforderlich.

\section{Interessenkonflikt}

Die Autorinnen/Autoren geben an, dass kein Interessenkonflikt besteht. 
Literatur

[1] Schwartz LH, Ozsahin M, Zhang GN et al. Synchronous and metachronous head and neck carcinomas. Cancer 1994; 74: 1933-1938. doi: doi:10.1002/1097-0142(19941001)74:7<1933::AIDCNCR2820740718>3.0.CO;2-X

[2] Zhai C, Cai Y, Lou F et al. Multiple Primary Malignant Tumors - A Clinical Analysis of 15321 Patients with Malignancies at a Single Center in China. J Cancer 2018; 9: 2795-2801. doi:10.7150/jca.25482

[3] Scherubl H, von Lampe B, Faiss S et al. Screening for oesophageal neoplasia in patients with head and neck cancer. British journal of cancer 2002; 86: 239-243. doi:10.1038/sj.bjc.6600018

[4] Leipzig B, Zellmer JE, Klug D. The role of endoscopy in evaluating patients with head and neck cancer. A multi-institutional prospective study. Archives of otolaryngology (Chicago, Ill: 1960) 1985; 111: 589594

[5] Stoeckli S], Zimmermann R, Schmid S. Role of routine panendoscopy in cancer of the upper aerodigestive tract. Otolaryngol Head Neck Surg 2001; 124: 208-212. doi:10.1067/mhn.2001.112311

[6] Okumura T, Aruga $\mathrm{H}$, Inohara $\mathrm{H}$ et al. Endoscopic examination of the upper gastrointestinal tract for the presence of second primary cancers in head and neck cancer patients. Acta Otolaryngol Suppl 1993; 501: 103-106

[7] Su YY, Chen WC, Chuang HC et al. Effect of routine esophageal screening in patients with head and neck cancer. JAMA otolaryngology - head \& neck surgery 2013; 139: 350-354. doi:10.1001/jamaoto.2013.46

[8] Hung SH, Tsai MC, Liu TC et al. Routine endoscopy for esophageal cancer is suggestive for patients with oral, oropharyngeal and hypopharyngeal cancer. PLoS One 2013; 8: e72097. doi:10.1371/journal.pone.0072097

[9] Chow TL, Lee DTY, Choi CY et al. Prediction of Simultaneous Esophageal Lesions in Head and Neck Squamous Cell Carcinoma: A Multivariate Analysis. Archives of Otolaryngology - Head \& Neck Surgery 2009; 135 : 882-885. doi:10.1001/archoto.2009.105

[10] Daniel M, Kamani T, Nogueira C et al. Perforation after rigid pharyngooesophagoscopy: when do symptoms and signs develop? The Journal of Laryngology \& Otology 2009; 124: 171-174. doi:10.1017| S0022215109991629

[11] Plott E, Jones D, McDermott D et al. A state-of-the-art review of esophageal trauma: where do we stand? Dis Esophagus 2007; 20: 279-289. doi:10.1111/j.1442-2050.2007.00714.x

[12] Weaver A, Fleming SM, Knechtges TC et al. Triple endoscopy: a neglected essential in head and neck cancer. Surgery 1979; 86: 493-496

[13] Cohn AM, Peppard SB. Multiple primary malignant tumors of the head and neck. Am J Otolaryngol 1980; 1: 411-417. doi:10.1016/s01960709(80)80022-6

[14] Shapshay SM, Hong WK, Fried MP et al. Simultaneous carcinomas of the esophagus and upper aerodigestive tract. Otolaryngol Head Neck Surg 1980; 88: 373-377. doi:10.1177/019459988008800410

[15] Gluckman JL, Crissman JD, Donegan JO. Multicentric squamous-cell carcinoma of the upper aerodigestive tract. Head Neck Surg 1980; 3 : 90-96

[16] Maisel RH, Vermeersch H. Panendoscopy for second primaries in head and neck cancer. The Annals of otology, rhinology, and laryngology 1981; 90: 460-464. doi:10.1177/000348948109000509

[17] McGuirt WF. Panendoscopy as a screening examination for simultaneous primary tumors in head and neck cancer: a prospective sequential study and review of the literature. Laryngoscope 1982; 92: 569-576

[18] McGuirt WF, Matthews B, Koufman JA. Multiple simultaneous tumors in patients with head and neck cancer: a prospective, sequential panendoscopic study. Cancer 1982; 50: 1195-1199. doi:10.1002/10970142(19820915)50:6<1195::aid-cncr2820500629>3.0.co;2-0
[19] Grossman TW, Toohill R], Lehman RH et al. Role of esophagoscopy in the evaluation of patients with head and neck carcinoma. Ann Otol Rhinol Laryngol 1983; 92: 369-372. doi:10.1177/000348948309200415

[20] Atkins JP Jr, Keane WM, Young KA et al. Value of panendoscopy in determination of second primary cancer. A study of 451 cases of head and neck cancer. Arch Otolaryngol 1984; 110: 533-534

[21] Schuller DE, Fritsch MH. An assessment of the value of triple endoscopy in the evaluation of head and neck cancer patients. Journal of surgical oncology 1986; 32: 156-158

[22] Grossman TW, Kita MS, Toohill R]. The diagnostic accuracy of pharyngoesophagram compared to esophagoscopy in patients with head and neck cancer. Laryngoscope 1987; 97: 1030-1032

[23] Parker JT, Hill JH. Panendoscopy in screening for synchronous primary malignancies. Laryngoscope 1988; 98: 147-149. doi:10.1288/ 00005537-198802000-00005

[24] Shaha A, Hoover E, Marti J et al. Is routine triple endoscopy cost-effective in head and neck cancer? American journal of surgery 1988; 155: 750753. doi:10.1016/s0002-9610(88)80036-9

[25] Abemayor E, Moore DM, Hanson DG. Identification of synchronous esophageal tumors in patients with head and neck cancer. J Surg Oncol 1988; 38: 94-96

[26] Grossman TW. The incidence and diagnosis of secondary esophageal carcinoma in the head and neck cancer patient. Laryngoscope 1989; 99: 1052-1056. doi:10.1288/00005537-198210000-00015

[27] Atabek U, Mohit-Tabatabai MA, Rush BF et al. Impact of esophageal screening in patients with head and neck cancer. Am Surg 1990; 56: 289-292

[28] Davidson J, Gilbert R, Irish J et al. The role of panendoscopy in the management of mucosal head and neck malignancy-a prospective evaluation. Head \& neck 2000; 22: 449-454; discussion 454-445

[29] Hashimoto CL, Iriya K, Baba ER et al. Lugol's dye spray chromoendoscopy establishes early diagnosis of esophageal cancer in patients with primary head and neck cancer. The American journal of gastroenterology 2005; 100: 275-282. doi:10.1111/j.1572-0241.2005.30189.x

[30] Tsao G], Damrose E]. Complications of esophagoscopy in an academic training program. Otolaryngology - head and neck surgery: official journal of American Academy of Otolaryngology-Head and Neck Surgery 2010; 142: 500-504. doi:10.1016/j.otohns.2010.01.008

[31] Rodriguez-Bruno K, Ali M], Wang SJ. Role of panendoscopy to identify synchronous second primary malignancies in patients with oral cavity and oropharyngeal squamous cell carcinoma. Head Neck 2011; 33: 949-953. doi:10.1002/hed.21561

[32] McGarey PO Jr, O'Rourke AK, Owen SR et al. Rigid Esophagoscopy for Head and Neck Cancer Staging and the Incidence of Synchronous Esophageal Malignant Neoplasms. JAMA Otolaryngol Head Neck Surg 2016; 142: 40-45. doi:10.1001/jamaoto.2015.2815

[33] Contini S, Consigli GF, Di Lecce F et al. Vital staining of oesophagus in patients with head and neck cancer: still a worthwhile procedure. Ital J Gastroenterol 1991; 23: 5-8

[34] Dhooge IJ, De Vos M, Albers FW et al. Panendoscopy as a screening procedure for simultaneous primary tumors in head and neck cancer. Eur Arch Otorhinolaryngol 1996; 253: 319-324

[35] Cianfriglia F, Di Gregorio DA, Manieri A. Multiple primary tumours in patients with oral squamous cell carcinoma. Oral Oncol 1999; 35: 157163. doi:10.1016/s1368-8375(98)00105-5

[36] Guardiola E, Pivot X, Dassonville O et al. Is routine triple endoscopy for head and neck carcinoma patients necessary in light of a negative chest computed tomography scan? Cancer 2004; 101: 2028-2033. doi:10.1002/cncr.20623

[37] Hujala K, Sipila J, Grenman R. Panendoscopy and synchronous second primary tumors in head and neck cancer patients. European archives of oto-rhino-laryngology: official journal of the European Federation of Oto-Rhino-Laryngological Societies (EUFOS): affiliated with the German 
Society for Oto-Rhino-Laryngology - Head and Neck Surgery 2005; 262: 17-20. doi:10.1007/s00405-004-0743-y

[38] Moschler O, Spahn TW, Middelberg-Bisping C et al. Chromoendoscopy is a valuable tool for screening of high-risk patients with head and neck cancer for early detection of esophageal cancer. Digestion 2006; 73 : 160-166. doi:10.1159/000094523

[39] Carvalho R, Areia M, Brito D et al. Diagnostic accuracy of lugol chromoendoscopy in the oesophagus in patients with head and neck cancer. Rev Esp Enferm Dig 2013; 105: 79-83

[40] Koo K, Harris R, Wiesenfeld D et al. A role for panendoscopy? Second primary tumour in early stage squamous cell carcinoma of the oral tongue. The Journal of laryngology and otology 2015; 129 (Suppl. 1): S27S31. doi:10.1017/s0022215114002989

[41] Ina H, Shibuya $\mathrm{H}$, Ohashi I et al. The frequency of a concomitant early esophageal cancer in male patients with oral and oropharyngeal cancer. Screening results using Lugol dye endoscopy. Cancer 1994; 73: 20382041. doi:10.1002/1097-0142(19940415)73:8<2038::aidcncr2820730804>3.0.co;2-x

[42] Muto M, Hironaka S, Nakane M et al. Association of multiple Lugol-voiding lesions with synchronous and metachronous esophageal squamous cell carcinoma in patients with head and neck cancer. Gastrointestinal endoscopy 2002; 56: 517-521. doi:10.1067/mge.2002.128104

[43] Fukuhara T, Hiyama T, Tanaka S et al. Characteristics of esophageal squamous cell carcinomas and lugol-voiding lesions in patients with head and neck squamous cell carcinoma. Journal of clinical gastroenterology 2010; 44: e27-e33. doi:10.1097/MCG.0b013e3181b31325

[44] Morimoto M, Nishiyama K, Nakamura S et al. Significance of endoscopic screening and endoscopic resection for esophageal cancer in patients with hypopharyngeal cancer. Jpn J Clin Oncol 2010; 40: 938-943. doi:10.1093/jjco/hyq068

[45] Lee CT, Chang CY, Lee YC et al. Narrow-band imaging with magnifying endoscopy for the screening of esophageal cancer in patients with primary head and neck cancers. Endoscopy 2010; 42: 613-619. doi:10.1055/s-0030-1255514

[46] Chung CS, Liao LJ, Lo WC et al. Risk factors for second primary neoplasia of esophagus in newly diagnosed head and neck cancer patients: a casecontrol study. BMC gastroenterology 2013; 13: 154. doi:10.1186/1471230x-13-154

[47] Kim DH, Gong EJ, Jung HY et al. Clinical significance of intensive endoscopic screening for synchronous esophageal neoplasm in patients with head and neck squamous cell carcinoma. Scand J Gastroenterol 2014; 49: 1486-1492. doi:10.3109/00365521.2013.832369

[48] Laohawiriyakamol S, Sunpaweravong S, Leelamanit V et al. Evaluating synchronous esophageal cancer in head and neck cancer patients using Lugol dye chromoendoscopy. J Med Assoc Thai 2014; 97: 1164-1170

[49] Krishnatreya M, Rahman T, Kataki AC et al. Synchronous primary cancers in the head and neck region and upper aero digestive tract: Role of triple endoscopy. Indian J Cancer 2015; 52: 53-56. doi:10.4103/0019509x. 175560

[50] Gong E], Kim DH, Ahn JY et al. Routine endoscopic screening for synchronous esophageal neoplasm in patients with head and neck squamous cell carcinoma: a prospective study. Dis Esophagus 2016; 29: $752-$ 759. doi: $10.1111 /$ dote. 12404

[51] Huang YC, Lee YC, Tseng PH et al. Regular screening of esophageal cancer for 248 newly diagnosed hypopharyngeal squamous cell carcinoma by unsedated transnasal esophagogastroduodenoscopy. Oral oncology 2016; 55: 55-60. doi:10.1016/j.oraloncology.2016.01.008

[52] Ni XG, Zhang QQ, Zhu JQ et al. Hypopharyngeal cancer associated with synchronous oesophageal cancer: risk factors and benefits of image-enhanced endoscopic screening. The Journal of laryngology and otology 2018; 132: 154-161. doi:10.1017/s0022215117002493

[53] Mohan M, Jagannathan N. Oral field cancerization: an update on current concepts. Oncol Rev 2014; 8: 244-244. doi:10.4081/oncol.2014.244

[54] Slaughter DP, Southwick HW, Smejkal W. "Field cancerization” in oral stratified squamous epithelium. Clinical implications of multicentric origin. Cancer 1953; 6: 963-968. doi:10.1002/10970142(195309)6:5<963::AID-CNCR2820060515>3.0.CO;2-Q

[55] Liu X, Wang X, Lin S et al. Dietary patterns and the risk of esophageal squamous cell carcinoma: A population-based case\&\#x2013;control study in a rural population. Clinical Nutrition 2017; 36: 260-266. doi:10.1016/j.clnu.2015.11.009

[56] Kuhlin B, Kramer B, Nefas V et al. Indicators for secondary carcinoma in head and neck cancer patients following curative therapy: A retrospective clinical study. Molecular and clinical oncology 2020; 12: 403410. doi:10.3892/mco.2020.2004

[57] Su YY, Chen WC, Chuang HC et al. Effect of Routine Esophageal Screening in Patients With Head and Neck Cancer. JAMA Otolaryngology Head \& Neck Surgery 2013; 139: 350-354. doi:10.1001/jamaoto. 2013.46

[58] Ritchie AJ, McGuigan J, McManus K et al. Diagnostic rigid and flexible oesophagoscopy in carcinoma of the oesophagus: a comparison. Thorax 1993; 48: 115-118. doi:10.1136/thx.48.2.115

[59] Deutsche Gesellschaft für Hals- Nasen-Ohrenheilkunde KH-C. AWMF S1Leitlinie 017/060: Ösophagoskopie. In; aktueller Stand: 06/2015

[60] Strobel K, Haerle SK, Stoeckli S] et al. Head and neck squamous cell carcinoma (HNSCC) - detection of synchronous primaries with 18F-FDGPET/CT. European Journal of Nuclear Medicine and Molecular Imaging 2009; 36: 919-927. doi:10.1007/s00259-009-1064-6

[61] Haerle SK, Strobel K, Hany TF et al. 18F-FDG-PET/CT versus panendoscopy for the detection of synchronous second primary tumors in patients with head and neck squamous cell carcinoma. Head \& Neck 2010; 32: 319-325. doi:10.1002/hed.21184 